\title{
Recent Results and Future Challenges for the NIST Charged-Capacitor Experiment
}

\author{
Neil M. Zimmerman, Jonathan L. Cobb, and Alan F. Clark
}

\begin{abstract}
This paper reports on recent results in some of the work toward developing a new capacitance standard using single electron tunneling (SET) devices. In particular, we plan on using a SET pump to charge a cryogenic standard capacitor and measure the voltage that develops. In this paper, we summarize: 1) measurements of the ratio of two capacitors in a bridge configuration, using a SET transistor as the null detector and 2) stability and leakage measurements on the cryogenic capacitors. We then discuss in detail several of the possible challenges, including the effects of stray capacitance and line impedance, and resulting requirements on the sensitivity of the SET null detector.
\end{abstract}

\section{INTRODUCTION AND MOTIVATION}

$\mathbf{T}$ HE CURRENT NIST effort in using single-electron tunneling (SET) devices for metrology follows from a proposal outlined by Williams, Ghosh, and Martinis [1]. It is predicated on the ability of the SET pump to transfer charge in single quanta, and thus to provide a fundamental current standard. The proposal is based on a simple idea: that one can use a small current to charge a small capacitor to a large voltage in a relatively short time. In particular, a $1 \mathrm{pA}$ current can charge a $1 \mathrm{pF}$ capacitor to $1 \mathrm{~V}$ in just $1 \mathrm{~s}$.

The implementation of this simple concept reduces to several important tasks, including the development of a singleelectron pump with a metrologically low level of errors (transferring $10^{8}$ electrons with an uncertainty of plus-orminus one), the use of a SET transistor as the null detector, and the development of cryogenic capacitors with very low loss. The first and second items (in particular, the SET pump) have been extensively investigated by NIST, Boulder, with very recent results essentially at the required low error rate [2], [3]. The second and third items have been in development at NIST, Gaithersburg in recent years. This has primarily been done by measuring ratios of two capacitors, using a SET transistor as the null detector in a bridge configuration [4], as well as measurements of the upper bound for the dissipation in the capacitors [5]. We will summarize recent results on these bridge and leakage measurements.

We will then analyze in some detail other topics relevant to the use of the cryogenic capacitors as a standard, particularly with respect to the comparison with room-temperature standards. These topics include minimizing stray capacitance (and the effect on sensitivity and accuracy), the effect of line

Manuscript received June 20, 1996; revised October 1, 1996.

The authors are with the National Institute of Standards and Technology, Electricity Division, Electronics and Electrical Engineering Laboratory, Technology Administration, U.S. Department of Commerce, Gaithersburg, MD 20899 USA (e-mail: neilz@eeel.nist.gov; URL: http://www.eeel.nist.gov/811/femg/set.html).

Publisher Item Identifier S 0018-9456(97)02150-5. impedance, and the comparison of using a SET transistor versus conventional electronics as the null detector.

\section{SuMmary OF RESUlts ON BRIDGE AND LEAKAGE MEASUREMENT}

We have conducted bridge measurements using two custommade capacitors and a SET transistor as the null detector [4]. We have made major improvements over previous attempts, by virtue of two changes, the first by using vacuum-gap capacitors, as opposed to the silica-based capacitors used earlier [6]. This change eliminated problems seen earlier, including a large ac signal out of phase with the capacitive response, and substantial dissipation (parallel leakage resistance of order $10^{13} \Omega$ ) [6].

The second improvement is in using on-chip coupling (gate) capacitors, $C_{\mathrm{C}}$, of substantially larger size than those used earlier, which were less than $0.1 \mathrm{fF}$. Currently, we are using values $C_{\mathrm{C}} \approx 0.5 \mathrm{fF}$. We have found that values larger than this $(1.0 \mathrm{fF})$ caused a large reduction in the modulation current, due to thermal smearing.

With these improvements, we previously demonstrated bridge measurements of the ratio of two vacuum-gap capacitors (each $0.5 \mathrm{pF}$ ), with an imprecision of about $3 \times 10^{-6}$, at a measurement frequency of about $300 \mathrm{~Hz}$ [4]. We have more recently performed a new set of measurements of this type, after improving our room-temperature circuit and the data-logging apparatus. Although the SET transistor used in this experiment was significantly noisier than in the previous experiment (see below) we were able to achieve the same level of imprecision (same experimental parameters). In this case, the major source of uncertainty was the discrete time-dependent random switching of the island charge (charge offset noise).

We have also conducted extensive measurements of the stability and frequency dependence of the capacitance ratio and the dissipation of the cryogenic capacitors, at frequencies in the audio range. Details may be found elsewhere [5]. In general, they are quite promising: from $100 \mathrm{~Hz}$ to $1000 \mathrm{~Hz}$, the upper bound on the change in the ratio was $5 \times 10^{-6}$. At the same audio frequencies, the long-term drift of the capacitance ratio had an upper bound of $1 \times 10^{-8} \mathrm{~h}^{-1}$. Finally, the dissipation was also immeasurably small, with a lower bound for the parallel leakage resistance of $1 \times 10^{19} \Omega$.

\section{EFFECTS OF STRAY CAPACITANCE}

We analyze in a simple model the effects of stray capacitance to ground on the sensitivity of both phases of the pump-capacitor experiment, considering the use of both 


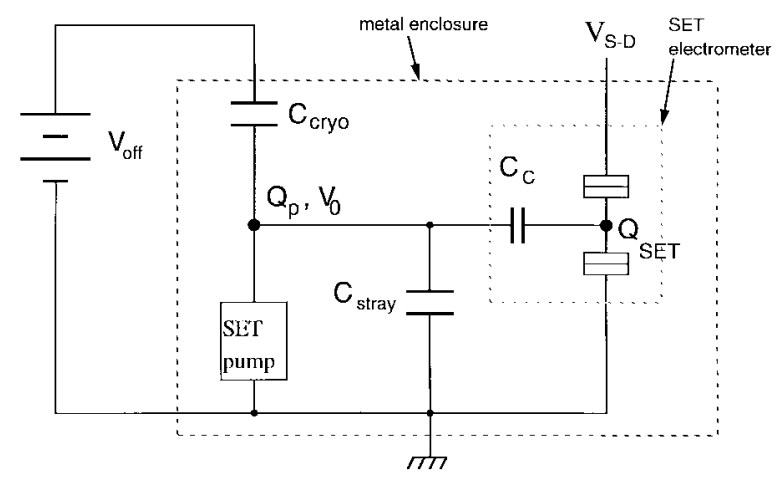

(a)

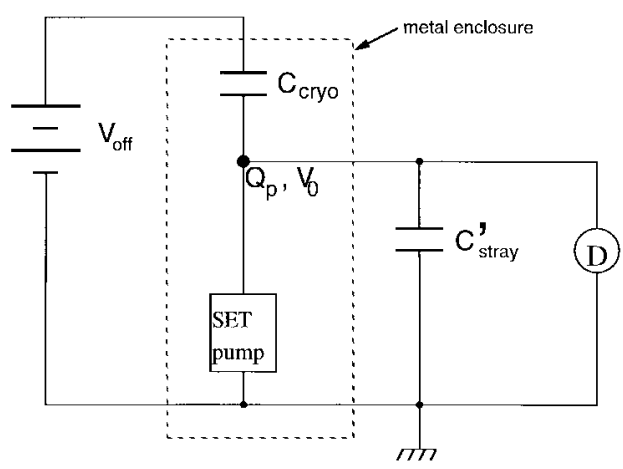

(b)

Fig. 1. Circuit diagram for the pump phase of the experiment, using a (a) SET electrometer or (b) conventional electrometer as the null detector, in order to assess the effect of stray capacitance on the detector sensitivity. Symbols are defined in the text.

conventional room-temperature as well as SET transistor null detectors.

In the first phase, the SET pump will be used to charge the capacitor (illustrated in Fig. 1 for both types of null detectors). In Fig. 1(a), we have the SET pump delivering charge $Q_{\mathrm{p}}$ to the center point, which develops a voltage $V_{0}$. This charge gets distributed to the three capacitors $C_{\text {cryo }}, C_{\text {stray }}$ and $C_{\mathrm{C}}$. To avoid a net voltage from developing across the pump, a feedback circuit (not shown) delivers a voltage $V_{\text {off }}$ to the outer plate of the cryogenic capacitor, $C_{\text {cryo }}$. This feedback circuit uses the SET electrometer as the null detector to minimize $V_{0}$ (virtual null). All the elements inside the larger dotted box are at low temperatures; more importantly, they are all within a metal enclosure, and spaced no more than a few $\mathrm{cm}$ apart. This geometry limits the important stray capacitance to ground, $C_{\text {stray }}$, to a fairly small value (as low as $5 \mathrm{pF}$ ) [6]. For comparison, in Fig. 1(b), we have the same circuit, except that the null detector, $D$, is a conventional room-temperature electrometer. This implies that the stray capacitance to ground, $C_{\text {stray }}^{\prime}$, has a much larger value (of order several hundred $\mathrm{pF}$ ) due to the long cable length. We note that the case in Fig. 1(b) is for comparative purposes only, since the leakage in the cables and the electrometer is many orders of magnitude higher than acceptable for the pump phase of this experiment.

We will now calculate the sensitivities of the null detection for this charged-capacitor phase, taking into account the stray capacitances to ground. For a perfect null detector, we would have $V_{0}=0$, and thus $V_{\text {off }}=Q_{\mathrm{p}} / C_{\text {cryo }}$ (neglecting the asynchronous voltages across the SET electrometer tunnel junction). With $V_{\text {off }}$ measured, and $Q_{\mathrm{p}}$ determined by counting electrons delivered through the pump, we could accurately determine $C_{\text {cryo }}$. The uncertainty in the measurement arises in the minimum resolvable voltage, $V_{0}$; as we shall see, the uncertainty is amplified by larger values of the stray capacitance to ground. We thus calculate the difference between $V_{\text {off }}$ and $Q_{\mathrm{p}} / C_{\text {cryo }}$, including the dependence on the stray capacitance.

For the case where the SET electrometer is used as null detector [Fig. 1(a)], we have the charge delivered by the pump distributing on the three capacitors

$$
Q_{\mathrm{p}}=Q_{\text {cryo }}+Q_{\text {stray }}+Q_{\mathrm{C}}
$$

Adding voltages around the left-hand loop, we have

$$
V_{\mathrm{off}}=Q_{\mathrm{cryo}} / C_{\mathrm{cryo}}-V_{0} \text {. }
$$

After some manipulation, and neglecting the capacitance in the lower tunnel junction of the SET electrometer (because it is of order $C_{\mathrm{C}}$, and both are much smaller than $C_{\text {stray }}$ and $\left.C_{\text {cryo }}\right)$, we have

$$
\left|V_{\text {off }}-Q_{\mathrm{p}} / C_{\text {cryo }}\right|=\left[\left(C_{\text {stray }}+C_{\mathrm{C}}\right) / C_{\text {cryo }}+1\right] V_{0} .
$$

Now, the charge on the island of the SET transistor is $Q_{\mathrm{SET}}=$ $C_{\mathrm{C}} V_{0}$; at the null condition, the minimum resolvable charge on the island is $Q_{\mathrm{SET}}=\delta Q_{\mathrm{SET}}$ (noise floor). Thus, the approximate (unsigned) offset between the feedback voltage and the voltage across the cryogenic capacitor, in terms of the minimum resolvable signal, $\delta Q_{\mathrm{SET}}$, is

$$
\left|V_{\text {off }}-\frac{Q_{\mathrm{p}}}{C_{\text {cryo }}}\right|=\frac{C_{\text {stray }}+C_{\mathrm{C}}+C_{\text {cryo }}}{C_{\text {cryo }}} \frac{\delta Q_{\mathrm{SET}}}{C_{\mathrm{C}}} .
$$

We note that, as expected, the presence of $C_{\text {stray }}$ increases the error due to $\delta Q_{\mathrm{SET}}$; however, since $C_{\text {stray }}$ and $C_{\text {cryo }}$ are of comparable magnitude, the increase is by a factor less than ten.

We now do a similar analysis for the Fig. 1(b); here the null detector is a conventional electrometer. At the null condition, we define $V_{0}=\delta V_{0}$, the minimum electrometer signal. In a similar analysis to the above, we derive

$$
\left|V_{\text {off }}-\frac{Q_{\mathrm{p}}}{C_{\text {cryo }}}\right|=\frac{C_{\text {stray }}^{\prime}+C_{\text {cryo }}}{C_{\text {cryo }}} \delta V_{0} .
$$

Here, in contrast to (1), the large value of $C_{\text {stray }}^{\prime}$ increases the uncertainty by a larger factor of about 100 .

In Fig. 2, we have the circuit for the second phase of the experiment, which is a standard bridge measurement comparing the cryogenic capacitor to the room-temperature standard, $C_{\text {stand }}$. Here, when the bridge is in balance, there is no excess charge, $Q_{b}$, or voltage, $V_{0}$, at the center point. Again, we examine both possible choices of null detector, a SET electrometer [Fig. 2(a)] and a conventional electrometer [Fig. 2(b)]. In both of these cases, the appropriate value for the capacitance to ground is the larger $C_{\text {stray }}^{\prime}$. Also, in contrast to the above, the conventional electrometer is an experimentally relevant choice for the null detector, because the much higher 


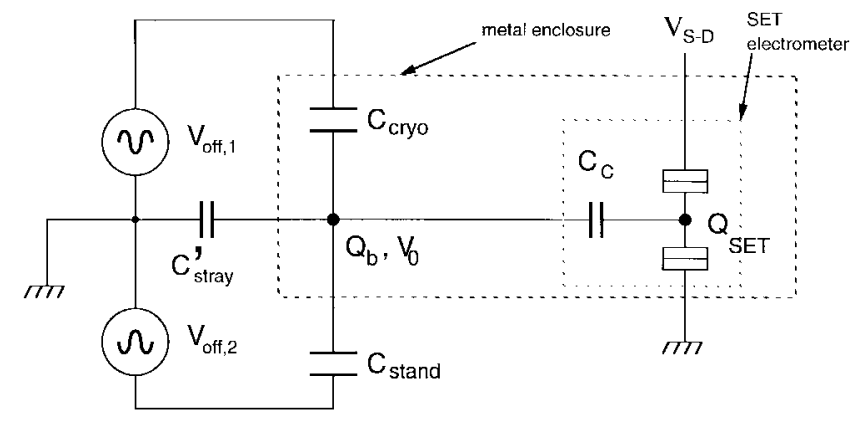

(a)

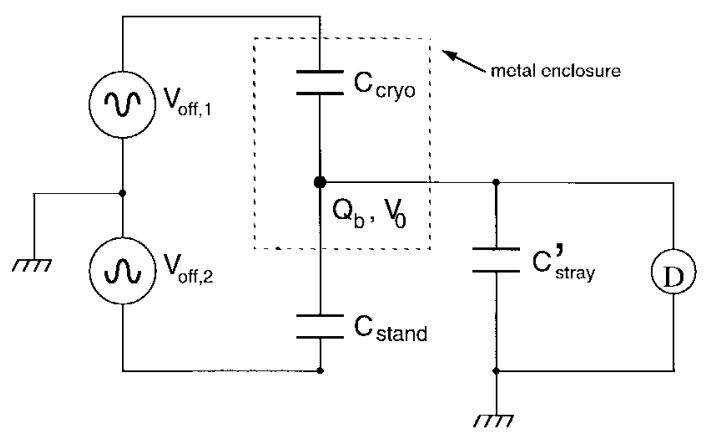

(b)

Fig. 2. Circuit diagram for the bridge phase of the experiment, with (a) SET electrometer or (b) conventional electrometer as the null detector.

frequency for the bridge measurement allows a higher leakage through the detector.

For the case of the bridge measurement, reasoning similarly to the above, a perfect null detector would imply that $V_{\mathrm{off}, 1} C_{\mathrm{cryo}}=V_{\mathrm{off}, 2} C_{\mathrm{stand}}$; thus the knowledge of the two AC voltages $V_{\text {off, } 1}$ and $V_{\text {off,2 }}$ (in practice, produced by a single source and a voltage divider) determines the ratio of the two capacitors. Thus, the relevant quantity to consider for imperfect null detectors is $V_{\text {off }, 1} C_{\text {cryo }}-V_{\text {off }, 2} C_{\text {stand }}$.

Using the SET electrometer [Fig. 2(a)], proceeding similarly to the above analysis, we derive

$$
V_{\text {off }, 1} C_{\text {cryo }}-V_{\text {off }, 2} C_{\text {stand }}=Q_{b}
$$

or

$$
\left|V_{\text {off }, 1} C_{\text {cryo }}-V_{\text {off }, 2} C_{\text {stand }}\right|=\frac{C_{\text {stray }}^{\prime}+C_{\mathrm{C}}}{C_{\mathrm{C}}} \delta Q_{\mathrm{SET}} .
$$

Here, we see that the decrease in the sensitivity due to the stray capacitance to ground is quite large $\left(C_{\mathrm{stray}}^{\prime} / C_{\mathrm{C}}\right.$, of order $\left.10^{5}\right)$.

Lastly, we have the case in Fig. 2(b), of a conventional null detector for the bridge measurement. Here, we simply have

$$
\left|V_{\text {off }, 1} C_{\text {cryo }}-V_{\text {off }, 2} C_{\text {stand }}\right|=Q_{b}=C_{\text {stray }}^{\prime} \delta V_{0} .
$$

With the previous results, we can approximate the uncertainties in the pump and bridge measurements. We use the following values: $C_{\text {stand }}=C_{\text {cryo }}=1 \mathrm{pF}, C_{\mathrm{C}}=1 \mathrm{fF}$; $V_{\text {off }}=10 \mathrm{~V}, V_{\text {off }, 1}=V_{\text {off }, 2}=100 \mathrm{~V} ; C_{\text {stray }}=5 \mathrm{pF}$, $C_{\text {stray }}^{\prime}=200 \mathrm{pF}$. For a conventional electrometer, we can estimate the voltage noise at audio frequencies (i.e., above the knee of the amplifier $1 / f$ noise) at $2 \mathrm{nV} / \mathrm{Hz}^{1 / 2}$. Thus, in a 0.1 $\mathrm{Hz}$ bandwidth at audio frequencies (i.e., using an AC lock-in

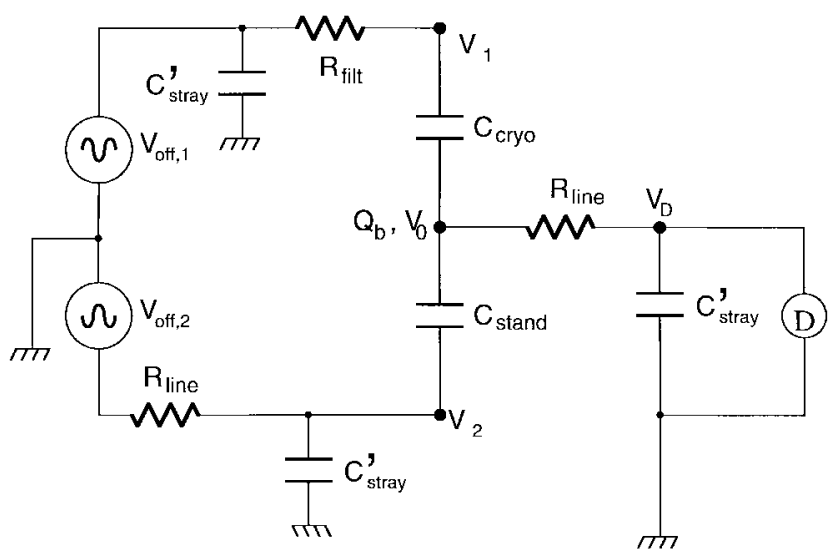

Fig. 3. Circuit diagram for the bridge phase of the experiment, with a conventional null detector. Here the various possible line impedances $R_{\text {filt }}$ and $R_{\text {line }}$ are explicitly considered.

measurement at $\omega=10^{4} \mathrm{~Hz}$ ), we have $\delta V_{0}=0.7 \times 10^{-9} \mathrm{~V}$. For a SET electrometer with charge noise of $10^{-4} \mathrm{e} / \mathrm{Hz}^{1 / 2}$ at $10 \mathrm{~Hz}$ or $10^{-3} \mathrm{e}^{-\mathrm{Hz}^{1 / 2}}$ at $0.1 \mathrm{~Hz}$, we have $\delta Q_{\mathrm{SET}}=$ $3 \times 10^{-4} \mathrm{e}=5 \times 10^{-23} \mathrm{C}$.

The resulting uncertainties are listed in Table I. We can see that the SET and conventional electrometers are comparable as the null detector for the pump phase (however, as noted earlier, only the SET electrometer has the necessary low leakage). We can also see that for the bridge phase, a conventional electrometer (with $2 \mathrm{nV} / \mathrm{Hz}^{1 / 2}$ noise) is preferable for the null detector. In both cases the estimates of the uncertainties, including the effect of stray capacitances, are probably low enough to allow metrological accuracy. We note that this analysis suggests that the pump phase, using the SET electrometer, has a predicted relative uncertainty somewhat larger than $10^{-8}$, even though we have used an estimate for the noise of the electrometer $\left(10^{-4} \mathrm{e} / \mathrm{Hz}^{1 / 2}\right.$ at $\left.10 \mathrm{~Hz}\right)$ which is among the best of the reported values [7]. This suggests, as we noted above, that optimizing the noise performance of the SET electrometer is an important topic for the ultimate success of this experiment.

\section{EFFECT OF LINE IMPEDANCE}

Here we will consider the effects of two types of line impedance, as indicated in Fig. 3. The first, $R_{\mathrm{filt}}$, approximates the discrete filters used to shield SET devices from high-frequency noise [8], and represents a resistance located close to the experiment; this leads us to lump the various capacitances to ground as being before $R_{\mathrm{filt}}$. The second, $R_{\text {line }}$, approximates the distributed line resistance; thus, we model the stray capacitance as being between the impedance and the capacitor or the detector. We have only considered the worst case: the bridge measurement using a conventional electrometer. Here, the null detector is physically far from the capacitors, which are physically far from the voltage source. Note that in all three cases, we have used the large value $C_{\text {stray }}^{\prime}$ for the stray capacitance. For simplicity, we have put only one of the impedances in the upper and lower loops of the bridge circuit; adding the others (e.g., $R_{\text {filt }}$ next to $C_{\text {stand }}$ ) complicates the analysis but sheds no additional light. 
TABLE I

List of Uncertainties for Both Experimental Phases, and Both Types of Null Detectors, Taking Into Account Stray Capacitance to Ground

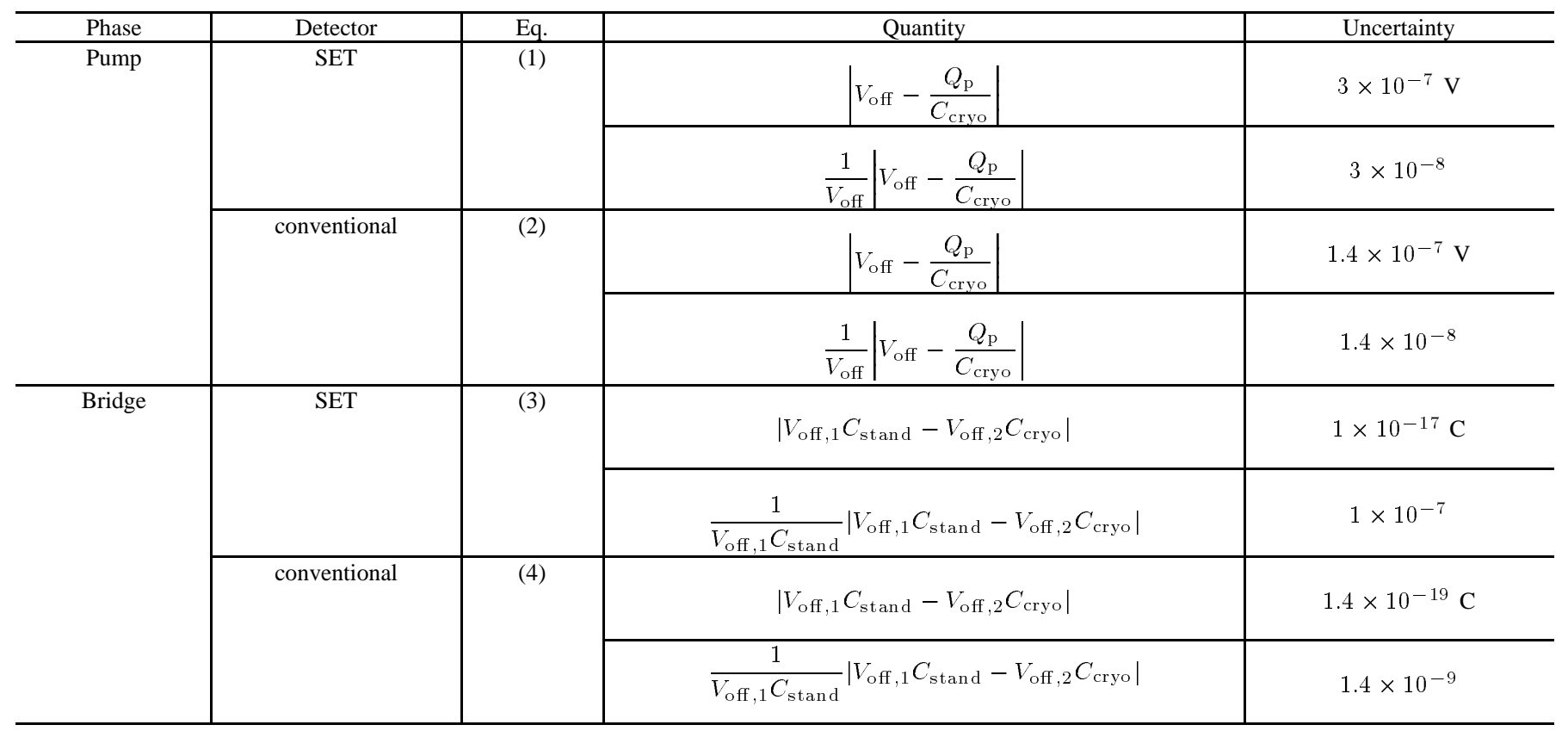

The deleterious effect of these line resistances is that the voltages applied to the capacitors $C_{\text {cryo }}$ and $C_{\text {stand }}$ will be different from $V_{\text {off,1 }}$ and $V_{\text {off,2 }}$, due to the voltage drops in the resistances. We wish to calculate these voltage drops. The three approximations we make are: 1) at virtual null, $\left.V_{0} \ll V_{\text {off }, 1}, V_{\text {off }, 2} ; 2\right) C_{\text {stray }}^{\prime} \gg C_{\text {stand }} ;$ and 3) $R_{\text {line }} \ll$ $\left.\left.1 / \omega C_{\text {stray }}^{\prime}\right), R_{\text {filt }} \ll 1 / \omega C_{\text {cryo }}\right)$ at $\omega=10^{4} s^{-1}$. For the top loop, the magnitude of the current flowing through $R_{\mathrm{filt}}$ is approximately

$$
I=C_{\text {cryo }} \dot{V}_{\text {off }, 1}=C_{\text {cryo }} \omega V_{\text {off }, 1}
$$

and thus the relative (unsigned) uncertainty in the voltages is

$$
\left|\frac{V_{1}-V_{\mathrm{off}, 1}}{V_{\mathrm{off}, 1}}\right|=R_{\mathrm{filt}} C_{\mathrm{cryo}} \omega .
$$

Note that the stray capacitance not between the resistance and the load does not enter. Similarly, for the lower loop, we derive

$$
\left|\frac{V_{2}-V_{\text {off }, 2}}{V_{\text {off }, 2}}\right|=R_{\text {line }} C_{\text {stray }}^{\prime} \omega \text {. }
$$

For a frequency $\omega=10^{4} \mathrm{~s}^{-1}$, to keep these relative uncertainties less than $10^{-8}$ requires $R_{\text {filt }}<1 \Omega$ and $R_{\text {line }}<0.01 \Omega$. This suggests that the meander-line type filters [8], which had DC resistances of about $1 \mathrm{k} \Omega$, are not usable; however, the simpler filters made from a coiled wire in a metal powder do have the low resistance necessary.

Finally, for the effect of the resistance in the line to the null detector, we similarly derive

$$
I=C_{\text {stray }}^{\prime} \dot{V}_{0}=C_{\text {stray }}^{\prime} \omega V_{0}
$$

and thus

$$
\left|\frac{V_{D}-V_{0}}{V_{0}}\right|=R_{\text {line }} C_{\text {stray }}^{\prime} \omega
$$

In this case, we require only that this relative error be much less than one, achievable if $R_{\text {line }}<1 \mathrm{M} \Omega$. As expected, the fact that the line to the null detector is at a virtual null minimizes the effect of line resistances.

\section{CONCLUSION}

We have made substantial progress in the fabrication of cryogenic capacitors, and in demonstrating the use of SET electrometers as capacitance bridge null detectors, in preparation for the SET pump-charged capacitor experiment. The results, outlined above, are detailed in two recent publications [4], [5].

In this paper, we have analyzed the effects of stray capacitance and line impedance. We have shown that, accounting for stray capacitances to ground and with reasonable noise performances, null detection in the pump phase can be satisfactorily achieved with a SET electrometer, and in the bridge phase with a conventional electrometer. As we have noted, this will also require SET electrometers with superior noise performance. We will also need to minimize the stray capacitance (to less than $5 \mathrm{pF}$ ) for the low-temperature end of the experiment, and to avoid excess line impedances caused by filters for the SET devices.

The authors gratefully acknowledge the wise guidance and suggestions of E. R. Williams, and the pioneering experimental work of R. Ghosh, as well as helpful discussions with M. W. Keller and J. M. Martinis.

\section{REFERENCES}

[1] E. R. Williams, R. N. Ghosh, and J. M. Martinis, J. Res. NIST 97, vol. 299, 1992.

[2] J. M. Martinis, M. Nahum, and H. D. Jensen, Phys. Rev. Lett., vol. 72, p. 904,1994

[3] M. W. Keller, J. M. Martinis, N. M. Zimmerman, and A. H. Steinbach, Appl. Phys. Lett., vol. 69, p. 1804, 1996. 
[4] A. F. Clark et al., Appl. Phys. Lett., vol. 66, p. 2588, 1995.

[5] N. M. Zimmerman, IEEE Trans. Instrum. Meas., vol. 45, p. 841, 1996.

[6] R. N. Ghosh, A. F. Clark, B. A. Sanborn, and E. R. Williams, Coulomb and Interference Effects in Small Electronic Structures. Gif-sur-Yvette, France: Frontier Editions, 1995, p. 173

[7] G. Zimmerli, T. M. Eiles, R. L. Kautz, and J. M. Martinis, Appl. Phys. Lett., vol. 61, p. 237, 1992

[8] D. Vion et al., J. Appl. Phys., vol. 77, p. 2519, 1995.

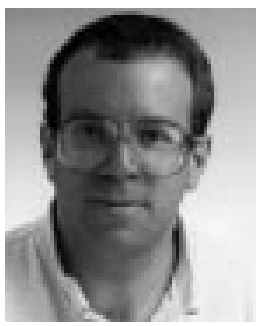

Neil M. Zimmerman was born on Long Island, NY, on June 21, 1960. He received the B.S. degree in physics from Rensselaer Polytechnic Institute, Troy, NY in 1982, and the M.S. and Ph.D. degrees in physics from Cornell University, Ithaca, NY in 1989.

He worked as a Postdoctoral Associate at AT\&T Bell Labs from 1989 to 1992, and as a Research Physicist at the Naval Research Lab from 1992 to 1994. He is currently employed as a Research Physicist in the Fundamental Electrical Measurements Group of the National Institute of Standards and Technology, Gaithersburg, $\mathrm{MD}$, where he works on applications of single electron tunneling devices for standards of electrical capacitance or current.

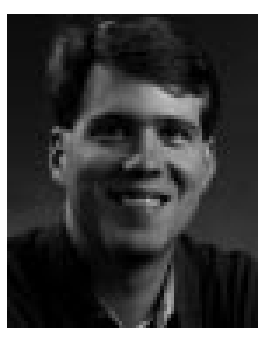

Jonathan L. Cobb was born in Bartlesville, OK, on June 1, 1967. He received the B.A. degree in physics from Baylor University, Waco, TX in 1989, and the Ph.D. degree in physics from the University of Texas at Austin, in 1995.

At the University of Texas, he studied bulk magnetic properties of high temperature superconductors. At present, he is a National Research Council Postdoctoral Research Associate in the Fundamental Electrical Measurements Group of the National Institute of Standards and Technology, Gaithersburg, MD. His primary research interest is electrical noise in single electron tunneling devices.

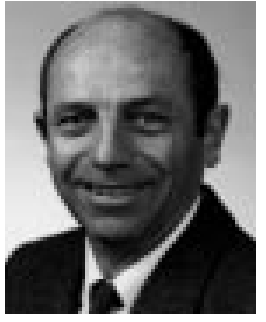

Alan F. Clark is Leader of the Fundamental Electrical Measurements Group for the National Institute of Standards and Technology, Gaithersburg, MD. In the past, he has been a Senior Research Physicis for the National Institute of Standards and Technology heading the Josephson voltage standards and single electron tunneling research efforts. He has also been Group Leader of the Superconductor and Magnetic Measurements Group at the Institute's Boulder, CO laboratory. Recently, he spent one and a half years as Liaison Scientist for the U.S Office of Naval Research-Europe, based in London, coordinating international science collaborations on superconductivity throughout Europe. He has been in low temperature physics research for more than 25 years, the last twenty in superconductivity, and has produced over 150 scientific publications. $\mathrm{He}$ has edited and contributed to many books on the low temperature properties of materials, superconductivity, and cryogenics, was Editor for the international journal Cryogenics, and is presently Editor-in-Chief of the IEEE TRanSACtions on APPLIED SUPERCONDUCTIVITY

Dr. Clark is a Fellow of the American Physical Society. 